\title{
Effect of Aspirin on Pre-eclampsia and Fetal Growth Restriction in Obese Pregnant Women: A Randomized Controlled Clinical Trial
}

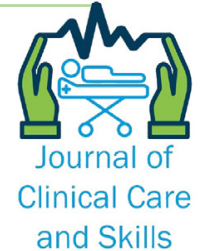

and Skills

\section{ARTICLE INFO}

\section{Article Type}

Original Research

\section{Authors}

Vanda R. ${ }^{1} M D$,

Aramesh Sh. ${ }^{1} M D$,

Sadeghian M. ${ }^{2} M D$,

Ghatei M.A. ${ }^{3} P h D$,

Ghaffari P.*1 $M D$

How to cite this article
Vanda R, Aramesh Sh, Sadeghian M,
Ghatei M.A, Ghaffari P. Effect of
Aspir-in on Pre-eclampsia and Fe-
tal Growth Restriction in Obese
Pregnant Women: A Randomized
Controlled Clinical Trial. Journal of
Clinical Care and Skills.
2020;1(4):159-164.

${ }^{1}$ Gynecology \& Obstetrics Department, Yasuj University of Medical Sciences, Yasuj, Iran

${ }^{2}$ Gynecology \& Obstetrics Department, Valiasr Hospital, Bafgh, Iran

${ }^{3}$ Cellular \& Molecular Research Center, Yasuj University of Medical Sciences, Yasuj, Iran

\section{*Correspondence}

Address: Gynecology \& Obstetrics Department, Medicine School, Yasuj University of Medical Sciences, Shahid Ghorbanali Jalil Street, Yasuj, Iran.

Phone: +98 (74) 33222756

Fax: +98 (74) 33222756

pari.ghaffari@gmail.com

\section{Article History}

Received: November 17, 2019

Accepted: January 06, 2020

ePublished: October 01, 2020

\section{A B S T R A C T}

Aims Obesity in pregnant women is a serious health problem that has many complications such as pre-eclampsia and fetal growth restriction (FGR). The aim of the present study was to evaluate the effect of aspirin on prevalence of pre-eclampsia and FGR in obese pregnant women.

Materials \& Methods This randomized controlled clinical trial was conducted on 187 pregnant women referring to birth center of Mofatteh Clinic, Yasuj University of Medical Sciences, Yasuj, Iran in 2017-2018.

The subjects were selected using purposive sampling method and randomly divided into two groups; intervention group $(n=89)$ and control group $(n=98)$. The intervention group received $80 \mathrm{mg} /$ day aspirin and the control group received placebo at 13-20 weeks of gestation until 34 weeks of pregnancy. Data were analyzed by SPSS 21 software using Chi square test. Findings The prevalence of pre-eclampsia (1.1 vs. 11.2) and FGR (3.4 vs. 21.4) were significantly lower in the intervention group compared to the control group $(\mathrm{p}<0.05)$. Conclusion Administration of $80 \mathrm{mg} /$ daily of aspirin from 13-20 weeks of gestation in obese pregnant women reduces the incidence of pre-eclampsia and FGR, which could support the positive role of aspirin in preventing pre-eclampsia and FGR.

Keywords Aspirin; Obesity; Pregnancy; Pre-eclampsia; Fetal Growth Restriction

\section{I T A T I O N L I N K S}

[1] Obesity: the prevention, identification, assessment and ... [2] Prevalence of obesity and trends in the distribution of body ... [3] Obesity in pregnancy: Complications and maternal ... [4] Prevalence and trends in prepregnancy normal ... [5] Prevalence of obesity, central obesity and the associated ... [6] Obesity: preventing and managing the global ... [7] Altered maternal and placental lipid metabolism ... [8] Obesity and pregnancy: mechanisms of short ... [9] Maternal outcomes in pregnancies complicated ... [10] Low-dose aspirin use for the prevention of ... [11] Prevalence of maternal obesity in an urban ... [12] Maternal obesity and risk of preter ... [13] Obesity and altered aspirin ... [14] Prevention of perinatal death and adverse perinatal ... [15] Aspirin versus placebo in pregnancies at ... [16] Aspirin for the prevention of preterm and term ... [17] Low-dose aspirin for prevention of adverse outcomes ... [18] The role of aspirin dose on the prevention of preeclampsia ... [19] Bilateral notching of uterine arteries at 12-14 weeks ... [20] Hypertension in pregnancy: the NICE ... [21] Usefulness of aspirin therapy in high-risk ... [22] Executive summary: American college ... [23] Preeclampsia ... [24] Comparing pregnancy, childbirth, and neonatal ... [25] Williams ... [26] ECPPA: randomised trial of low dose aspirin ... [27] Acetylsalicylic acid for the prevention of preeclampsia ... [28] 0C08.05: Impact of 1st trimester aspirin on population ... [29] Low dose acetylsalicylic acid in prevention of ... [30] Influence of maternal obesity on fetal growth at different ... [31] Prevention of preeclampsia and intrauterine growth ... [32] A prospective management study of slow-release ... [33] Evaluation of the effect of low doses aspirin in prevention ... [34] Randomized controlled trial using lowdose aspirin in ... [35] Prevention of preeclampsia with low-dose aspirin: a systematic ... 


\section{Introduction}

Maternal obesity is one of the most common risk factors in pregnancy care [1]. The prevalence of obesity in women of childbearing age and pregnant women largely depends on the used definition, the characteristics of the study population, and the increased prevalence of obesity in the general population [2]. In the United States, the National Center for Health Statistics in 2011-2014 found that $34 \%$ of women aged $20-39$ were obese (BMI $\geq 30$ ) [3]. Among American women in 2015, the results show that $26 \%$ were obese and $26 \%$ were overweight [4]. Overall prevalence rate of $18.8 \%$ for obesity and $34.8 \%$ for overweight in population of Iranian aged 20-70 years who are living in north of Iran was reported [5]. According to World Health Organization (WHO) report, obesity has become epidemic in the world [6].

Adipose tissue is an active endocrine organ and, when excessively available, can disrupt the metabolic, vascular, and especially inflammatory, regulation of many organs during pregnancy and affect pregnancy outcomes [7]. For example, insulin resistance associated with obesity and disorders of the inflammatory pathway can affect the placental growth and function [8]. Maternal BMI has been increasingly reported as an independent risk factor for both pre-eclampsia and pregnancy hypertension [9]. Recent studies have shown that maternal obesity with a high-fat diet results in restricted intrauterine growth and less expression of placental fatty acid transporters [10]. The prevalence of gestational diabetes mellitus (GDM) in obese women is significantly higher than that of non-obese women [11]. A population-based cohort study in Sweden involving more than 1.5 million single-births confirmed overweight and obese women were at risk preterm births in all trimesters of pregnancy [12].

Obesity is an independent risk factor for atherosclerotic morbidity and cardiovascular mortality requiring antithrombotic therapies. Obesity is characterized by increased levels of coagulation factors, fibrin breakdown, increased platelet activity, insulin resistance, inflammation and endothelial dysfunction. Aspirin is an anti-platelet drug [13] whose mechanism of action is not yet known but may include improvements in the repair of the uterine spiral artery, which is usually defective in pre-eclampsia [14].

The optimal dose of aspirin to prevent pre-eclampsia is controversial. Dose differences are partly based on different compounds and doses available in different regions of the world. An experiment with $150 \mathrm{mg}$ of aspirin with a marked reduction in the risk of early pre-eclampsia was used [15]. A meta-analysis showed that aspirin reduced the risk of early pre-eclampsia but had no effect on late pre-eclampsia; this study states that the treatment was effective only when using aspirin began in gestational age less than 16 weeks with daily doses $\geq 100 \mathrm{mg}{ }^{[16]}$. Bujold et al. have also shown that if aspirin treatment is started at $\leq 16$ weeks of gestation, the risk of pre-eclampsia, fetal growth restriction, preterm labor, and perinatal mortality is significantly reduced, whereas the effect of treatment after 16 weeks, significantly less. In addition, the reduction in the risk of pre-eclampsia appears to be related to the aspirin dose; it's recommended the dose of $80 \mathrm{mg}$ daily should be considered for optimal benefits [17]. In a metaanalysis study by Roberge et al. a total of 45 randomized controlled trials involving a total of 20909 pregnant women randomly consumed between 50-150mg aspirin daily. When aspirin was started at 16 week of pregnancy, there was a significant decrease in the dose-response effect for the prevention of pre-eclampsia. Severe preeclampsia and fetal growth restriction (FGR) with higher doses of aspirin showed better results; thus, it is better identifying women at high risk of preeclampsia early in pregnancy and initiating aspirin use in the first 16 weeks of pregnancy [18].

Low doses of aspirin are recommended for women at high risk for pre-eclampsia, but there is no agreement on high risk criteria. Criteria stated by the United States Preventive Services Task Force (USPSTF) include a history of pre-eclampsia in previous pregnancies particularly with early onset and with adverse outcomes, multiple pregnancy, chronic hypertension, type 1 or 2 gestational diabetes, autoimmune disease (antiphospholipid and lupus syndrome). The USPSTF has also recommended women with several moderate factors (nulliparity, obesity, family history of pre-eclampsia in mother or sister, age 35 and older, geographic socioeconomic characteristics such as African-American or low social level), birth history of low birth weight or small for gestational age, adverse complications in previous pregnancy, pregnancy interval less than 10 years) are also considered to be high risk group and prophylaxis with aspirin [10, 19]. The WHO recommends that low-dose aspirin (75mg/day) be used for 12-20 weeks of pregnancy for women at high risk (history of pre-eclampsia, diabetes, chronic hypertension, autoimmune or kidney disease, or multiple pregnancies). But it's expressed there is limited evidence about the benefits of low-dose aspirin in other subgroups of high-risk women [20].

In Iran, obesity is a serious health problem that has many complications such as cardiovascular diseases, cancers and more. Considering obesity disorders in pregnant women; the occurrence of dangerous complications in the mother and fetus such as preeclampsia and FGR; contradictory results of previous studies on the usefulness of aspirin in obese individuals and reduction of pre-eclampsia and FGR in pregnant women; the presence of confounding 


\section{1}

factors in previous studies and the lack of a study that focused exclusively on obese pregnant women lacking other risk factors for pre-eclampsia and FGR, this study aimed to evaluate the effect of aspirin on prevalence of pre-eclampsia and FGR in obese pregnant women (without previous history of preeclampsia and hypertension). This would allow the design of appropriate interventions in the case of obesity or increased risk factors related to preeclampsia and FGR.

\section{Materials and Methods}

This single blind randomized controlled clinical trial was conducted on pregnant women referring to birth center of Mofatteh Clinic, Yasuj University of Medical Sciences, Yasuj, Iran in 2017-2018. Allocation to groups was done by "table of random numbers" method based on the Ebrashy et al. [21] and considering $10 \%$ of loss to follow up during sampling; at least 194 people were estimated for study. The subjects were selected using purposive sampling method according to the inclusion and exclusion criteria. Inclusion criteria were age 18-40, single pregnancy, the first gravid, BMI $\geq 30$, tends to participate in the study, non underlying known disease such as chronic hypertension, liver disease, kidney disease, diabetes mellitus, no using aspirin or allergy history, no ultrasound findings on NT e.g. mean PI UT $>1.5$ or PAPPA $<0.4$, no history of previous pre-eclampsia, no history of previous fetal growth restriction, normality of tests and screening.

Exclusion criteria included unwillingness to participate in the study, not available for evaluations, abortion and preterm delivery for reasons other than pre-eclampsia and FGR.

After the approval of the ethics committee of Yasuj medical university of sciences, Iran, this study was conducted and the purpose of the study explained to subjects. Written consent was received from all participants who were advised that they could withdraw at any time they wish during the study.

195 people were entered to the study and randomly divided into two groups; intervention group $(n=97)$ and control group $(n=98)$. Eight patients in the intervention group were excluded from the study, and thus 89 patients in this group were studied.

The intervention group received $80 \mathrm{mg} /$ day aspirin and the control group received placebo at 13-20 weeks of gestation until 34 weeks of pregnancy.

Studies suggest that trophoblastic attack on the spiral artery of the uterus usually begins about 8-10 weeks of gestation, ending at 16-18 weeks but may continue until 22 weeks. It is believed that interventions that can help to improve this process should probably start as early as 8 -10 weeks and end after 16-18 weeks, at the appropriate time [13]. On the other hand, there is no agreement on the optimal timing of aspirin withdrawal. Some researchers have suggested that aspirin should be discontinued at 36 weeks of
Vanda R. et al.

gestation or 5-10 days before possible delivery [22]; however, congenital or fetal adverse effects associated with use of low-dose aspirin at delivery has not been established [23]. Therefore, intervention in both groups in present study was started from 1320 weeks. The intervention group received aspirin 80 $\mathrm{mg} /$ day up to 34 weeks of gestation. The placebo group received empty capsules up to 34 weeks of gestation.

Body mass index (BMI), intrauterine growth restriction (IUGR), and pre-eclampsia of participants were detected as follows:

BMI:

Weight and height were calculated by weight/ height squared $\left(\mathrm{kg} / \mathrm{m}^{2}\right)$ in all patients.

\section{Diagnosis of IUGR:}

The first step in identifying IUGR is the evaluation of fetal growth by comparing the fetal parameters at a definite moment of pregnancy to the expected fetal parameters according to various referential for the respective gestational age. The most widely used definition of IUGR is a fetus whose estimated weight (EFW) is below the $10^{\text {th }}$ percentile for its gestational age. The next step after the determination of ineffective growth is to differentiate between a physiologically small fetus, small for gestational age fetus (SGA) and IUGR - which is pathological - with the use of additional tools like Doppler ultrasound sampling of fetal arterial or venous compartments, amniotic fluid volume assessment and other biophysical methods with the goal of indicating the actual state of the small fetus [24]. Diagnosis was based on following criteria in Doppler ultrasound sampling: EFW or $\mathrm{AC}<3^{\text {rd }}$ centile mean Uterine artery pulsatility index (UtA-PI) $>95^{\text {th }}$ centile decrease in EFW or AC $>2$ quartiles on growth centiles, Umbilical artery pulsatility index (UA-PI) $>95^{\text {th }}$ centile, Cerebroplacental ratio (CPR) $<5^{\text {th }}$ centile. All Doppler sonography was performed by one perinatologist and Ultrasound Machine GE Voluson 730 (GE Company; United State).

\section{Diagnosis of Pre-eclampsia:}

Blood pressure $\geq 90 / 140 \mathrm{~mm} \mathrm{Hg}$ after twenty weeks of gestation with proteinuria $\geq 300 \mathrm{mg}$ per 24 hours or $\geq 1$ in urine dipstick.

Data were analyzed by SPSS 21 software using Chi square test.

\section{Findings}

In total, 187 subjects participated in the study. There was no significant difference between the two groups in terms of the age, the time of using intervention and BMI (Table 1).

There was a significant difference between the two groups in the incidence of pre-eclampsia $(\mathrm{p}<0.001)$ and FGR $(p=0.005)$. In which, the incidence of preeclampsia and FGR were lower in aspirin group vs. placebo group (Table 2). 
Table 1) Distribution of absolute and relative frequency of demographic characteristics in intervention $(n=89)$ and control $(n=98)$ groups at the beginning of the study (the numbers in parentheses are percentages)

\begin{tabular}{|c|c|c|c|}
\hline Variables & $\begin{array}{c}\text { Intervention } \\
\text { group }\end{array}$ & $\begin{array}{l}\text { Control } \\
\text { group }\end{array}$ & P. value \\
\hline \multicolumn{4}{|l|}{ Age } \\
\hline $18-25$ years old & $23(25.8)$ & $25(25.5)$ & \multirow{2}{*}{0.95} \\
\hline $26-40$ years old & $66(74.2)$ & $73(74.5)$ & \\
\hline \multicolumn{4}{|c|}{ Start time of intervention } \\
\hline $\begin{array}{l}<16 \text { weeks of } \\
\text { pregnancy }\end{array}$ & $32(36.0)$ & $40(40.8)$ & \multirow{2}{*}{0.49} \\
\hline $\begin{array}{l}>16 \text { weeks of } \\
\text { pregnancy }\end{array}$ & $57(64.0)$ & $58(59.2)$ & \\
\hline \multicolumn{4}{|l|}{ BMI } \\
\hline $30-34.9 \mathrm{~kg} / \mathrm{m}^{2}$ & $60(67.4)$ & $82(83.7)$ & \multirow{2}{*}{0.11} \\
\hline $35-40 \mathrm{~kg} / \mathrm{m}^{2}$ & $29(32.6)$ & $16(16.3)$ & \\
\hline
\end{tabular}

Table 2) Distribution of absolute and relative frequency of preeclampsia and FGR in intervention $(n=89)$ and control $(n=98)$ groups after intervention (the numbers in parentheses are percentages)

\begin{tabular}{lccc}
\multicolumn{1}{c}{ Variables } & $\begin{array}{c}\text { Intervention } \\
\text { group }\end{array}$ & $\begin{array}{c}\text { Control } \\
\text { group }\end{array}$ & P. value \\
\hline $\begin{array}{l}\text { Pre-eclampsia } \\
\text { Positive }\end{array}$ & $3(3.4)$ & $21(21.4)$ & $<0.001$ \\
Negative & $86(96.6)$ & $77(78.6)$ & \\
FGR & $1(1.1)$ & $11(11.2)$ & 0.005 \\
Positive & $88(98.9)$ & $87(88.8)$ & \\
Negative & & & \\
\hline
\end{tabular}

\section{Discussion}

This study aimed to assess the effect of aspirin on pre-eclampsia and FGR in obese pregnant women compared to the control group. Results of the study showed a lower incidence of pre-eclampsia (1.1 vs. 11.2) and FGR (3.4 vs. 21.4) in aspirin compared to control group which reflects the positive impact of aspirin in lower incidence of FGR and pre-eclampsia in obese pregnant women.

Hypertension disorders that occur during pregnancy are common disorders and responsible for a large proportion of deaths and maternal complications [25]. There is no completely validated method for entirely detecting vasospasm and vascular involvement, but Doppler ultrasound as a noninvasive method is an appropriate test for predicting uterine vascular involvement. Low-dose aspirin is a drug that prevents or modifies the course of pre-eclampsia. Aspirin inhibits angiotensin II through inhibition of thromboxane A2 synthesis [25]. Although early successes have been reported with low doses of aspirin in suppressing thromboxane A2 and prostacyclin superiority, few clinical trials have reported the efficacy of aspirin [26]. In the metaanalysis of Bujold et al. [27], the efficacy of aspirin in weeks of less than 16, 16-19, and after 20 in high-risk women with abnormal Doppler was investigated in the follow-up of pre-eclampsia. Results showed that initiation of aspirin before 16 week of pregnancy significantly reduced the incidence of pre-eclampsia and blood pressure in pregnancy. Similar to our findings, the findings of Baschat et al. indicate that low-dose aspirin reduces placental blood flow resistance and prevents pre-eclampsia [28]. Similarly to our results in a clinical trial on 86 women to evaluate the effect of aspirin with dosing on the prevention of pregnancy hypertension in high-risk women who had abnormal findings at weeks 12-14 of gestational vaginal Doppler sonography, administration of $80 \mathrm{mg}$ of aspirin (43 women) and placebo (43 women) daily showed that aspirin use significantly reduced the incidence of gestational hypertension (11.6\% vs. $37.2 \%)^{[29]}$.

In a retrospective cohort study in Tokyo, Japan, Tanaka et al. [30] examined the effect of maternal obesity on fetal development during different gestation periods. Overall, this study showed that pre-pregnancy maternal obesity (pre-pregnancy BMI) affects pre-eclampsia and fetal growth restriction in the third trimester of pregnancy. Bujold et al. in a systematic and meta-analysis to evaluate the prevention of pre-eclampsia and intrauterine growth restriction with aspirin in early pregnancy, summarized 27 randomized controlled trial (RCT) studies that showed low-dose aspirin use before week 16 of gestation is associated with a significantly reduced incidence of severe pre-eclampsia, FGR, preterm labor, and all placental complications. They reported that low-dose aspirin administration before week 16 of gestation could prevent $50 \%$ and $55 \%$ of pre-eclampsia and FGR, respectively [31].

Ebrashy et al. performed an RCT study on the effect of low-dose aspirin in preventing pre-eclampsia and FGR in high-risk women with abnormal uterine Doppler findings. The study included 139 cases with a $35 \%$ incidence of pre-eclampsia and $62 \%$ in the control group and FGR occurred in 19\% of aspirin group and $32 \%$ of control group. They found that low doses of aspirin from 14-16 weeks in high risk preeclampsia patients with abnormal uterine Doppler reduces the incidence of pre-eclampsia, but its effect on reducing FGR needs further study [21]. In another study, 86 high-risk women with abnormal findings in their vaginal Doppler sonography at 12-14 weeks of gestation were studied and the results showed that aspirin reduced the incidence of pre-eclampsia by $11.6 \%$ vs. $37.2 \%$ in placebo group [29].

Contrary to the findings of our study and other similar studies referred to above; in Harrington et al. study [32] on 216 women who had been exposed to pre-eclampsia on the basis of ultrasound findings at weeks 17-23 of gestation; the results showed that there was no significant difference between the two groups in the incidence of pre-eclampsia, which may be due to the decrease in the incidence of preeclampsia in the aspirin-treated group in this study due to the higher gestational age (17-23 weeks). In this study, aspirin use has started later than our study. The findings of some recent studies show that the effect of low dose aspirin varies based on 
gestational age [31]. A study by Atarod et al. in Iran showed that low-dose aspirin use in primiparous women was not effective in preventing preeclampsia, which may be the reason for the difference in aspirin initiation at gestational age (13-32 week of pregnancy) [33]. In a study by YU et al., it was found that in women with abnormal Doppler findings, administration of aspirin after 23 weeks of gestation did not prevent the development of pre-eclampsia [34]. Another meta-analysis of 22 studies found that administration of aspirin in high-risk women had little effect on preventing pre-eclampsia. However, the risk factors for this study included high risk of pre-eclampsia in the mother, maternal illnesses, a history of pre-eclampsia in the individual, multiple pregnancies, abnormal Doppler findings, roll over test, and angiotensin sensitivity test that was different with our criteria, which included obesity and the absence of pre-eclampsia risk criteria [35].

However, the discrepancy between the findings in aspirin efficacy may be due to differences in gestational age at the time of initiation of aspirin use, studying on high or low risk women and different criteria for evaluation of risk differences in aspirin dose, inclusion criteria and sample size.

Although, this study has much strength, it is not without limitations. The participants were recruited from a public clinic of hospital; therefore result reduced the generalizability. Further studies with larger sample size recruited from a less homogenous population are recommended.

Given the contradictory results regarding the role of aspirin in the prevention of pre-eclampsia and FGR; a larger sample size study in high-risk mothers (multiple pregnancy, high age, and having chronic hypertension or diabetes) seems to be useful. If the positive role of aspirin is proven, it can be recommended to prescribe it at an early age in high risk pregnant women.

\section{Conclusion}

Administration of $80 \mathrm{mg} /$ daily of aspirin from $13-20$ weeks of gestation in obese pregnant women reduces the incidence of pre-eclampsia and FGR, which could support the positive role of aspirin in preventing preeclampsia and FGR.

Acknowledgements: The authors are grateful to Dr. Fatemeh Bazarganipour (Yasuj University of Medical Sciences) for editing the manuscript.

Ethical Permission: The Ethics Committee of the Yasuj University of Medical Sciences approved the study (IR.YUMS.REC.1397.152) and this study was registered in Iranian Registry of clinical trials (IRCT20160524028038N5). All women gave written informed consent.

Conflict of Interests: The authors declare no conflict of interests.

Authors' Contribution: Vanda R. (First author), Introduction author/ Original researcher (25\%); Aramesh S. (Second author), Assistant (10\%); Sadeghian M. (Third author), Assistant (20\%); Ghatei MA. (Fourth author), Methodologist/ Statistical analyst (10\%); Ghafari P. (fifth author), Introduction author/ Methodologist/ Original researcher/ Statistical analyst/ Discussion author (35\%)

Funding: The research grant provided by Research Deputy of Yasuj University of Medical Sciences (YUMS). The role of the funding body was collection and analysis.

\section{References}

1- NICE Clinical Guidelines. Obesity: the prevention, identification, assessment and management of overweight and obesity in adults and children [Internet]. London: National Institute for Health and Clinical Excellence (UK); 2006 [cited 2009 Dec 1]. Available from: https://www.ncbi.nlm.nih.gov/pubmed/22497033.

2- Flegal KM, Carroll MD, Kit BK, Ogden CL. Prevalence of obesity and trends in the distribution of body mass index among US adults, 1999-2010. JAMA. 2012;307(5):491-7.

3- Ramsey PS, Schenken RS, Pi-Sunyer FX. Obesity in pregnancy: Complications and maternal management [Internet]. Up To Date; 2017 [cited 2009 Dec 1]. Available from: https://www uptodate com/contents/obesityinpregnancy-complications-and-maternal-management.

4- Deputy NP, Dub B, Sharma AJ. Prevalence and trends in prepregnancy normal weight-48 states, New York City, and District of Columbia, 2011-2015. MMWR Morb Mortal Wkly Rep. 2018;66(51-52):1402-7.

5- Hajian-Tilaki KO, Heidari B. Prevalence of obesity, central obesity and the associated factors in urban population aged 20-70 years, in the north of Iran: a population-based study and regression approach. Obes Rev. 2007;8(1):3-10.

6- World Health Organization. Obesity: preventing and managing the global epidemic. Report of a WHO Consultation (WHO Technical Report Series 894) [Internet]. Geneva: WHO; 2000 [cited 2009 Dec 1]. Available from: https://www.who.int/nutrition/publications/obesity/WH 0_TRS_894/en/

7- Delhaes F, Giza SA, Koreman T, Eastabrook G, McKenzie CA, Bedell S, et al. Altered maternal and placental lipid metabolism and fetal fat development in obesity: Current knowledge and advances in non-invasive assessment. Placenta. 2018;69:118-24.

8- Catalano PM, Shankar K. Obesity and pregnancy: mechanisms of short term and long term adverse consequences for mother and child. BMJ. 2017;356:j1.

9- Robinson HE, O'connell CM, Joseph KS, McLeod NL. Maternal outcomes in pregnancies complicated by obesity. Obstet Gynecol. 2005;106(6):1357-64.

10- LeFevre ML. Low-dose aspirin use for the prevention of morbidity and mortality from preeclampsia: US Preventive Services Task Force recommendation statement. Ann Intern Med. 2014;161(11):819-26.

11- Ehrenberg HM, Dierker L, Milluzzi C, Mercer BM. Prevalence of maternal obesity in an urban center. Am J Obstet Gynecol. 2002;187(5):1189-93.

12- Cnattingius S, Villamor E, Johansson S, Bonamy AKE, Persson M, Wikström AK, et al. Maternal obesity and risk of preterm delivery. JAMA. 2013;309(22):2362-70.

13- Norgard NB. Obesity and altered aspirin pharmacology. Clin Pharmacokinet. 2018;57(6):663-72.

14- Roberge S, Nicolaides KH, Demers S, Villa P, Bujold E. Prevention of perinatal death and adverse perinatal outcome using low-dose aspirin: a meta-analysis. Ultrasound Obstet Gynecol. 2013;41(5):491-9. 
15- Rolnik DL, Wright D, Poon LC, O'gorman N, Syngelaki A, de Paco Matallana C, et al. Aspirin versus placebo in pregnancies at high risk for preterm preeclampsia. $\mathrm{N}$ Engl J Med. 2017;377(7):613-22.

16- Roberge S, Bujold E, Nicolaides KH. Aspirin for the prevention of preterm and term preeclampsia: systematic review and metaanalysis. Am J Obstet Gynecol. 2018;218(3):287-93. e1.

17- Bujold E, Roberge S, Nicolaides KH. Low-dose aspirin for prevention of adverse outcomes related to abnormal placentation. Prenat Diagn. 2014;34(7):642-8.

18- Roberge S, Nicolaides K, Demers S, Hyett J, Chaillet N, Bujold $\mathrm{E}$. The role of aspirin dose on the prevention of preeclampsia and fetal growth restriction: systematic review and meta-analysis. Am J Obstet Gynecol. 2017;216(2):110-20. e6.

19- Vainio M, Kujansuu E, Koivisto AM, Maenpaa J. Bilateral notching of uterine arteries at 12-14 weeks of gestation for prediction of hypertensive disorders of pregnancy. Acta Obstet Gynecol Scand. 2005;84(11):1062-7.

20- Redman CW. Hypertension in pregnancy: the NICE guidelines. Heart. 2011;97(23):1967-9.

21- Ebrashy A, Ibrahim M, Marzook A, Yousef D. Usefulness of aspirin therapy in high-risk pregnant women with abnormal uterine artery Doppler ultrasound at 14-16 weeks pregnancy: randomized controlled clinical trial. Croat Med J. 2005;46(5):826-31.

22- Hirsh J, Guyatt G, Albers GW, Harrington R, Schünemann HJ. Executive summary: American college of chest physicians evidence-based clinical practice guidelines. Chest. 2008;133(6 Suppl):71S-109S.

23- August P, Jeyabalan A. Preeclampsia: Prevention [Internet]. Up To Date; 2018 [cited 2009 Dec 1]. Available from:

https://www.uptodate.com/contents/preeclampsiaprevention.

24- Foroozanfard F, Asemi Z, Bazarganipour F, Taghavi SA, Allan H, Aramesh S. Comparing pregnancy, childbirth, and neonatal outcomes in women with different phenotypes of polycystic ovary syndrome and healthy women: a prospective cohort study. Gynecol Endocrinol. 2020;36(1):61-5.

25- Cunningham FG, Leveno KJ, Bloom SL, Hauth JC, Rouse DJ, Spong CY. Williams obstetrics. 23rd Edition. New York: McGraw Hill; 2010.

26- Group EC. ECPPA: randomised trial of low dose aspirin for the prevention of maternal and fetal complications in high risk pregnant women. $\mathrm{Br} \mathrm{J}$ Obstet Gynaecol. 1996;103(1):39-47.

27- Bujold E, Morency AM, Roberge S, Lacasse Y, Forest JC, Giguère Y. Acetylsalicylic acid for the prevention of preeclampsia and intra-uterine growth restriction in women with abnormal uterine artery Doppler: a systematic review and meta-analysis. J Obstet Gynaecol Can. 2009;31(9):818-26.

28- Baschat A, Poon LY, Blitzer M, Nicolaides K, Harman C. OC08.05: Impact of 1st trimester aspirin on population prevalence of pre-eclampsia. Ultrasound Obstet Gynecol. 2009;34(S1):14.

29- Vainio M, Kujansuu E, Iso-Mustajärvi M, Mäenpää J. Low dose acetylsalicylic acid in prevention of pregnancyinduced hypertension and intrauterine growth retardation in women with bilateral uterine artery notches. BJOG. 2002;109(2):161-7.

30- Tanaka K, Matsushima M, Izawa T, Furukawa S, Kobayashi Y, Iwashita M. Influence of maternal obesity on fetal growth at different periods of pregnancies with normal glucose tolerance. J Obstet Gynaecol Res. 2018;44(4):691-6.

31- Bujold E, Roberge S, Lacasse Y, Bureau M, Audibert F, Marcoux S, et al. Prevention of preeclampsia and intrauterine growth restriction with aspirin started in early pregnancy: a meta-analysis. Obstet Gynecol. 2010;116(2 Pt 1):402-14.

32- Harrington K, Kurdi W, Aquilina J, England P, Campbell S. A prospective management study of slow-release aspirin in the palliation of uteroplacental insufficiency predicted by uterine artery Doppler at 20 weeks. Ultrasound Obstet Gynecol. 2000;15(1):13-8.

33- Atarod Z, Rahman Pourzanjani H, Hosseinzadeh M, Khalilian AR. Evaluation of the effect of low doses aspirin in prevention of pre-eclampsia and primipara. J Mazandaran Univ Med Sci. 2003;13(39):36-40. [Persian]

34- Yu CK, Papageorghiou AT, Parra M, Dias RP, Nicolaides $\mathrm{KH}$. Randomized controlled trial using low-dose aspirin in the prevention of pre-eclampsia in women with abnormal uterine artery Doppler at 23 weeks' gestation. Ultrasound Obstet Gynecol. 2003;22(3):233-9.

35- Ruano R, Fontes RS, Zugaib M. Prevention of preeclampsia with low-dose aspirin: a systematic review and meta-analysis of the main randomized controlled trials. Clinics. 2005;60(5):407-14. 